\title{
Desktop Haptic Interface for Simulation of Hand-Tremor
}

\author{
Gastone Pietro Rosati Papini, Marco Fontana, and Massimo Bergamasco
}

\begin{abstract}
This paper presents a haptic system that is conceived to support the design process of a class of products or services in order to make them more accessible to people affected by hand tremor diseases. The main aim is to foster the designer empathy allowing her/him to directly feel the effect of the impairment in first person. Specifically, a desktop haptic device is employed to induce a programmable hand-tremor, that is typically observed in people affected by some kind of neurological diseases, on healthy subjects (i.e., the designers). The developed tool is based on a wrist-attached haptic interface with a workspace that is comparable to that of the arm of the user. Such device is able to exert controlled forces on the user's wrist and induces a hand-tremor whose frequency and amplitude are correlated with those measured on impaired people. The control of the device is based on a custom trajectory-tracking algorithm that takes as input tremor signals that are acquired on patients using an optical motion tracking system. In this paper, we present the employed haptic system, the structure of the control system and the experimental validation of the controller done through the acquisition of data on six patients affected by Parkinson's disease.
\end{abstract}

Index Terms-Machine haptics, on-line identification, empathy, grab, wrist

\section{INTRODUCTION}

$\mathrm{T}$ HE verification of usability and acceptance of end-users is a fundamental step in the design process of successful products/services. This particularly holds true for products or services that are characterised by having a strong component of physical interaction in their final use, such as: consumer electronic devices, furniture/office equipment, kitchen appliances, car interiors and computer-interfaces, to name a few.

In answer to this need, design practices have evolved toward a user-centred approach, in which product specifications are built around users' needs and requirements. In this context, recent methodologies propose a design approach based on the concept of empathy, borrowed from psychological science. Empathy is the capability of recognising emotions experienced by another subject and, in the context of industrial design, it means to get an understanding of the end-user point of view. The discipline of Empathic Design (EmD) was born with the aim of proposing a design methodology that elicits in the designer a deep understanding of the end-user perspective and her/his relation with the products.

Recent researches in the field of Engineering Design are investigating the possibility of applying EmD principles to Inclusive Design [1], i.e., design of systems that are accessible and usable to all, including special users such as the disabled or elderly. This means that specific procedures and tools have been conceived for generating in the designer a first-person understanding of the end-user disabilities.

- The authors are with the PERCRO Laboratory of TeCIP Institute, Scuola Superiore Sant' Anna, Pisa, Italy.

E-mail:\{g.rosatipapini,m.fontana,m.bergamasco\}@sssup.it.

Manuscript received 29 Nov. 2014; revised 19 Nov. 2015; accepted 23 Nov. 2015. Date of publication 3 Dec. 2015; date of current version 16 Mar. 2016.

Recommended for acceptance by M. Santello.

For information on obtaining reprints of this article, please send e-mail to: reprints@ieee.org, and reference the Digital Object Identifier below.

Digital Object Identifier no. 10.1109/TOH.2015.2504971
An example of this scenario is when a designer of furniture for an office, that has to be accessed by visually impaired users, is induced to feel the limitations of the end-user sight and adapt her/his design solutions accordingly.

Few examples of such EmD tools were previously developed. A first method for designer-empathising with disabled end-users has been proposed and validated by Cardoso and Clarkson [2]. The authors propose a set of hardware wearable devices, called "capability-loss simulators", that limit motion and perception capabilities of the designer in a way to temporarily simulate the end-user's reduced abilities. Similar concepts have been lately applied by car manufacturers and at MIT within the project AGNES (Age Gain Now Empathy System) where researchers have developed an ageing suit able to introduce several mobility and vision impediments [3]. These systems are able to effectively simulate several visual or motor impairments associated with ageing. However, due to their passive nature, they lack flexibility and require long times to be donned, set up and tuned.

With the aim of providing a more flexible and reprogrammable tool available for designer, researchers have started to investigate the possibility to simulate disabilities through computer based programmable Human Computer Interaction (HCI) systems. Their main goal is to develop programmable systems that enable the simulation of several types/severity of impairment that can be easily selected and set. Specifically, the simulation of disabilities has been studied for visual impairments in the field of web-design and word processor software, with the development of simple software tools in [4], [5] and of a dedicated system for the design of accessible user interfaces based on EmD principles in [6].

In this context, we propose a novel interactive application in which a kinesthetic haptic device is employed on able people to elicit sensations that are commonly felt by those affected by particular forms of neurological motor 


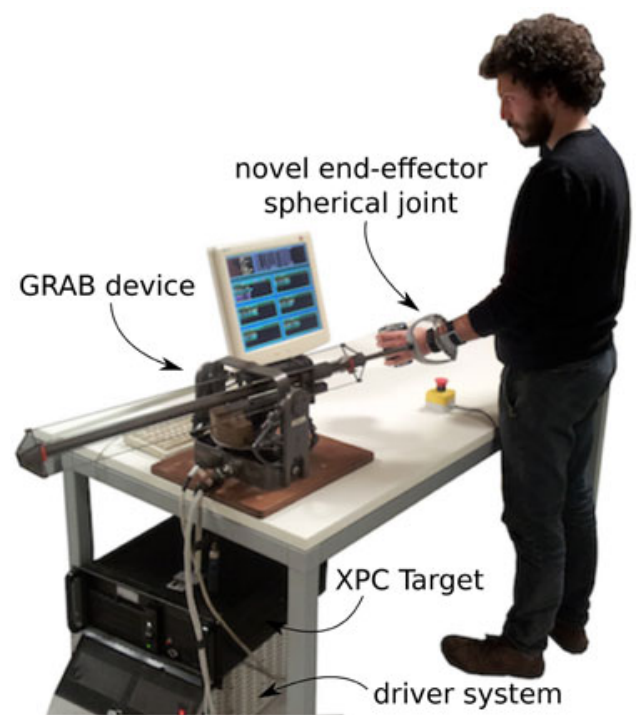

Fig. 1. Overall system employed for the generation of tremor composed by a haptic interface (GRAB device), controller, and a wrist attachment (novel end-effector spherical joint).

disabilities. Specifically, the system we have developed aims at simulating the experience of manipulating/handling objects when affected by hand-tremor due to neurological diseases. This tool is intended to be employed by designers of system/products that have to be accurately manipulated in their use, e.g., kitchen appliances, keyboards, devices operated by handles, hand-tools, glasses etc.

In order to induce tremor on a healthy subject, we use a 3 Degrees of Freedom (DoF) desktop haptic interface called GRAB [7]. The system, with its main components, is represented in Fig. 1. The user can stand in front of a table with the device attached to her/his forearm and is free to manipulate and explore objects. The control of the device is achieved through a recording and playback of tremoraffected trajectories acquired on people suffering from tremor diseases. The controller is designed in a way to leave the user free to make his intentional movements required for the task with a superimposed tremor disturbance.

The paper is structured as follows. The second section of the paper briefly introduces and qualitatively describes the hand-tremor pathologies. The third section presents the overall system employed to simulate hand-tremor and its experimental characterisation. The fourth section explains the procedure that was employed for acquiring and analysing hand-tremor data on a set of tremor-affected (Parkinsonians) patients. The fifth section shows the control strategy that was designed to generate hand-tremor. In the sixth section, we provide an experimental validation of the design controller.

The present paper is based on a previous work conducted by some of the authors of this paper reported in [8]. Part of Section 2 and part of Section 3.1 are taken from this previous work. The rest of the paper is dedicated to the presentation of new original contents.

\section{Hand-Tremor Simulation}

Tremor is a common disturbance of movement, and it is defined as a rhythmic and oscillatory movement of a body part, caused by involuntary repetitive muscle contractions
[9]. It can vary in frequency and amplitude, and is influenced by motor, physiological, and psychological factors. Tremor is most often noticed in hands and arms, but it may affect several parts of the body. Tremor can be classified as:

- Resting: tremor occurs when muscles are resting. The tremor can disappear or become less noticeable during movements. [10].

- Intention: tremor occurs at the end of a purposeful (intended) movement, such as writing, pressing a button, or reaching for an object. The tremor can disappear while the affected body part is at rest [11].

- Postural or actions: tremor occurs when holding the arm or leg against gravity or other forces in a position. This may happen when holding a cup, the arms out, or standing up straight [12].

Everyone has some tremor that superimposes on the voluntary movements of the limbs. Stress, fatigue, anger, fear, caffeine, and cigarettes may temporarily induce an increase of this type of "natural" tremor. However, there are several diseases whose symptoms consist of levels of tremor that influence and sometimes impede the normal execution of Activities of Daily Living (ADL). The three most diffused types of pathologies associated with relevant levels of handtremor are: Essential tremor, Parkinson and Cerebellar Tremor. A comprehensive study and survey of disease that are associated with tremor were conducted by Wyne in [13]. A numerical investigation of the spectral analysis of tremor is reported in [14].

Each disease is associated with distinct features of tremor such as typical bandwidth and amplitude of movements. However, the detailed characteristics of resting, intention and postural tremor may vary from patient to patient. Obviously, the simulation of all the aspects that are associated with tremor pathologies would be desirable but would require complex and expensive systems. In this work, we are focusing on postural tremor and its effects on manipulation/handling of objects. Thus, functionally we aim at developing a system able to induce in the user's hand a programmed vibration of a few millimetres in the frequency range of $4-12 \mathrm{~Hz}$ [13].

None of previous scientific works has addressed the simulation of a programmed hand-tremor. However, several researchers have investigated technical solutions for actively reducing hand-tremor. Avizzano and Bergamasco [15] developed a system for enabling people affected by tremor associated with Multiple Sclerosis to interact with a PC-based environment. Additionally, tremor damping techniques were employed for removing the natural tremor of healthy users to extend their abilities during very accurate manipulation of hand-held instruments [16], [17].

Simulating tremor on a healthy user with high fidelity is a very challenging goal. Hand-tremor is an extremely complex three dimensional phenomena than involves the musculoskeletal apparatus and the nervous system. The intensity, the main frequency and the complex 3D trajectories assumed by the limbs of the user are extremely variable upon the posture [18], type/intention of action and muscle activity [11], [19]. Moreover, hand tremor is often associated with other type of purposeless unwanted movements such as overshot or limits in speed and the range motion of limbs [11]. 


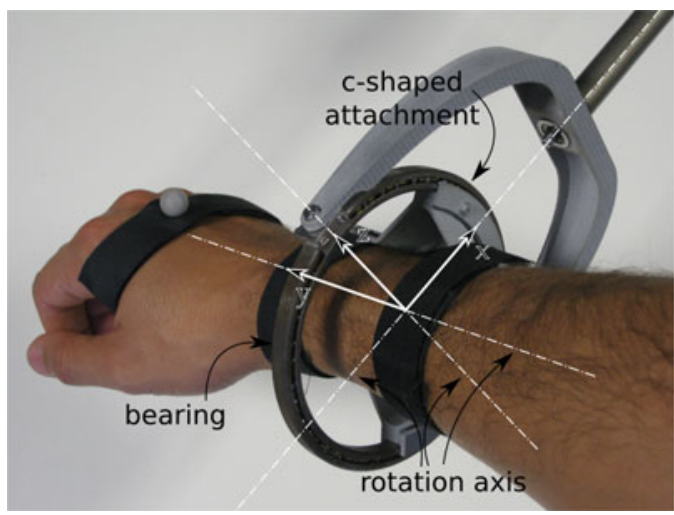

Fig. 2. Passive 3 DoF spherical joint implemented with rapid prototyping manufactured components and commercial slim-bearing.

A complete tremor simulation system should consider all these aspects simultaneously, resulting in a very complex device that adjusts its behaviour according to the posture of the user arm, the state of activation of muscles, intention of motion and should also consider physiological and environmental effects.

In this work we have assumed several simplifications (illustrated in the next section) in order to be able to effectively reproduce the effect of the impairment in limited operating conditions.

\section{System Description}

\subsection{Haptic Interface}

Different possible strategies and devices capable of introducing an artificial tremor on the hand of healthy users were analysed in [8]. Among the possible solutions, the employment of a three DoF haptic interface called GRAB [7] appeared to be the most suitable option.

The GRAB, developed by Scuola Superiore Sant'Anna, is a desktop force-feedback device based on a serial kinematic chain composed of two rotary and one prismatic joint, with a workspace that is comparable to that of a human arm. The actuators are grounded and steel cables are employed to transmit torque to joints. The device is able to exert continuous forces in a bandwidth of $40 \mathrm{~Hz}$ over any desired direction in a range of $4 \mathrm{~N}$ in the worst condition (typically 6$7 \mathrm{~N})$. All DoFs are sensorized with high resolution angular optical encoders which guarantee position resolution of $0.1 \mathrm{~mm}$ measured at the end-effector. Stiffness measured at the end-effector is typically in the range of $6-7 \mathrm{~N} / \mathrm{mm}$ and absolute force accuracy is in the range of $50-100 \mathrm{mN}$ [20].

The end-effector of GRAB was originally equipped with a small spherical joint that integrates a thimble for fingertip attachment or a sensorized plate that is employed when high force accuracy is required [21]. For the purpose of hand-tremor simulation we need the user to have his hands completely free to manipulate and explore surfaces and objects. For this reason, we designed a novel passive three DoF spherical joint whose last link is tightly fastened around the user's forearm, close to the wrist, using velcro strips (see Fig. 2). The joint is made of light-weight polyamide charged with aluminium powder, manufactured with a Direct Printing rapid prototyping system. The second of the three joints is implemented with a large diameter bearing
(Kaydon Reali-Slim Bearing K10008CP0) in order to place the centre of the spherical joint as close as possible to the axis of pronosupination motion of the forearm. An ergonomic c-shape attachment that envelops the forearm of the user was designed to have a comfortable and solid doublesupported connection.

The haptic interface is driven by a control unit with a closed loop sample rate of $2 \mathrm{kHz}$, that is equipped with encoder counters, Digital-to-Analog converters and current drivers.

The complete system (Fig. 1) is composed by:

- the GRAB with the novel developed wrist attachment;

- a current-driver box that includes the driving electronics DC $48 \mathrm{~V}-400 \mathrm{~W}$ power supply;

- a real-time operating system XPC target machine employed for implementation of the developed controllers.

The control is designed in a MATLAB/Simulink environment. No force sensors are employed and motor torques are directly estimated from the readings of the motor currents.

\subsection{System Model and Characterisation}

This section presents the procedure that we adopted for the identification of the dynamic properties of the overall mechanical system composed by the GRAB device attached to the forearm of a healthy subject (the user) through the novel end-effector.

An accurate characterisation of the dynamic parameters is required since the proposed system only makes use of the readings of angular sensors placed on the axis of the motors of the HI. Thus, in order to have a precise estimation of the movements of the user's wrist, the mechanical impedance between the position of the motors and the position of the user's wrist has to be characterised. An alternative option is to use additional systems (such as external optical/magnetic trackers) to have a direct measure of the actual position of the user's wrist. However, we decided to avoid the use of external measuring systems to maintain a minimal complexity, thus avoiding additional costs, setup time and increase the portability of the system.

The global dynamic system features a quite complex behaviour due to:

- large variability of dynamic parameters depending on the position (of both human arm and device);

- non-linearity given by friction forces, transmissions and backlash in the device;

- the variable impedance of human arm depending on the level of activation of muscles and posture.

In order to reduce the complexity of the problem, in this work, we have considered the following main assumptions:

(1) The position of the device during the operation (and during its characterisation) is kept in the neighbourhood of the centre of its workspace. In this position, the equations of motion of the device are highly simplified since the mass and the stiffness matrix are diagonal and fully isotropic.

(2) The user's arm assumes always the same (known) posture and its muscles are in a relaxed state. In 


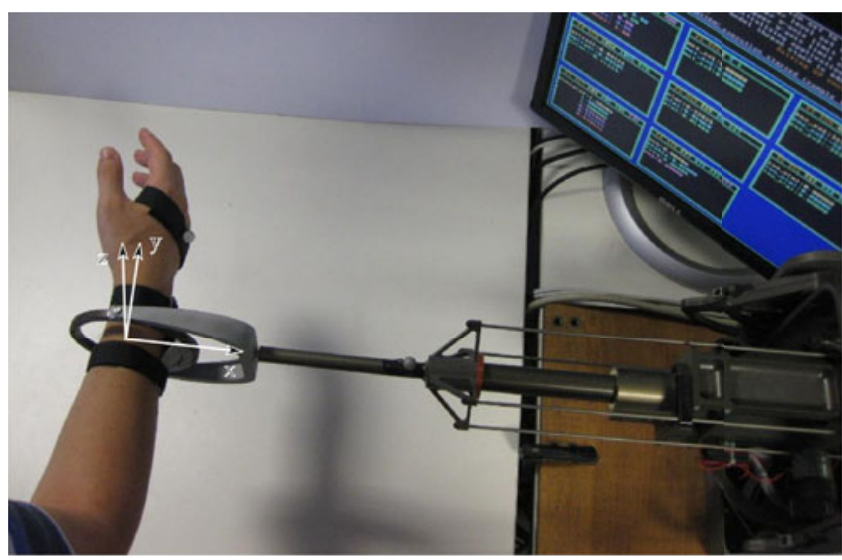

Fig. 3. The setup during the operation.

these conditions, the arm of the user is assumed to respond as a mass connected to the device through a contact impedance made of a damper-spring parallel. For simplicity, the contribution of user's arm stiffness and damping are not considered.

These may look as very strong assumptions but even with these limitations the developed tool could be employed in a variety of simple tests that include manipulative actions which require small displacements and forces such as: pushing a button, operating a selector, holding a pen, pointing toward a direction, etc.

The above-mentioned assumptions make it possible to employ a simplified dynamic model. Due to its isotropy in the considered position, the system is insensitive to the choice of the reference frame, thus, a Cartesian coordinate system is adopted (see Fig. 3 ).

The simplified model can be used for any of the considered directions; in Fig. 4 the $x$ direction is used for reference. The considered model includes the force of the motor $F_{x}$ that acts on the mass of the motor $m_{m}$, connected to the mass of the link $m_{l}$ through a transmission system that features a stiffness $k_{t}$ in parallel with a damping $c_{t}$. The human arm is modelled as a simple mass $m_{h}$ and the contact interface is assumed to be described by a contact stiffness $k_{c}$ in parallel with a contact damping $c_{c}$. All the above mentioned model parameters are reduced through kinematic relations to the Cartesian coordinates.

The identification of the system was conducted in the centre of the workspace. The GRAB was worn by a user having his forearm fixed in a reference position attached to an external grounded frame through appropriate fixing belts. In this condition, the displacement of $m_{h}$ is null, making it possible to identify the mechanical parameters of the device and of contact impedance in each direction (i.e., $m_{m}$, $m_{l}, k_{t}, c_{t}$ and $k_{c}$ and $c_{c}$ ). In this phase, we are not estimating the mass of the user $m_{h}$ since it is assumed to be variable with the user. This parameter is left unknown and is

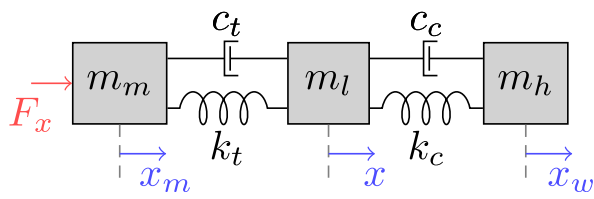

Fig. 4. Scheme of the assumed simplified dynamic model for the $x$ direction; parameters are considered reduced to the Cartesian directions.
TABLE 1

The Identified System Parameters in the Three Axes

\begin{tabular}{ccccccc}
\hline & $m_{l}(\mathrm{~kg})$ & $m_{m}(\mathrm{~kg})$ & $c_{c}\left(\frac{\mathrm{Ns}}{\mathrm{m}}\right)$ & $c_{t}\left(\frac{\mathrm{Ns}}{\mathrm{m}}\right)$ & $k_{c}\left(\frac{\mathrm{kN}}{\mathrm{m}}\right)$ & $k_{t}\left(\frac{\mathrm{kN}}{\mathrm{m}}\right)$ \\
\hline$x$ & 0.49 & 0.10 & 62.42 & 9.30 & 7.04 & 23.0 \\
$y$ & 0.26 & 0.10 & 9.16 & 0.76 & 2.26 & 2.35 \\
$z$ & 0.20 & 0.14 & 11.02 & 1.73 & 1.03 & 5.31 \\
\hline
\end{tabular}

evaluated through an online estimator during the operation of the controller (see Section 5).

A linear chirp signal with an intensity of $5 \mathrm{~N}$ with startingfinal frequency of $0.1-100 \mathrm{~Hz}$ was chosen in order to fully cover the frequency range of interest. The mechanical parameters were identified with an optimisation procedure that minimises the error between the frequency response of the numerical system and the response of the chosen model. The obtained values for these parameters are given in Table 1, while in Fig. 5, the measured (solid green line) and the modelled (dashed blue line) responses of the system are compared in Bode plots.

Specifically, the transfer functions

$$
\frac{x_{m}(s)}{F_{x}(s)}, \frac{y_{m}(s)}{F_{y}(s)}, \frac{z_{m}(s)}{F_{z}(s)},
$$

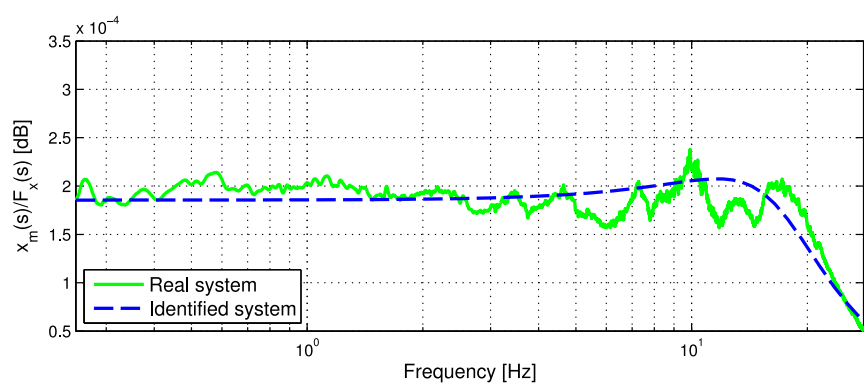

(a)

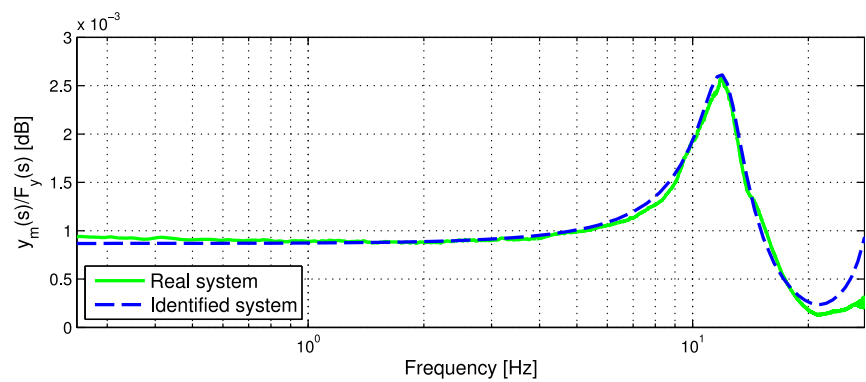

(b)

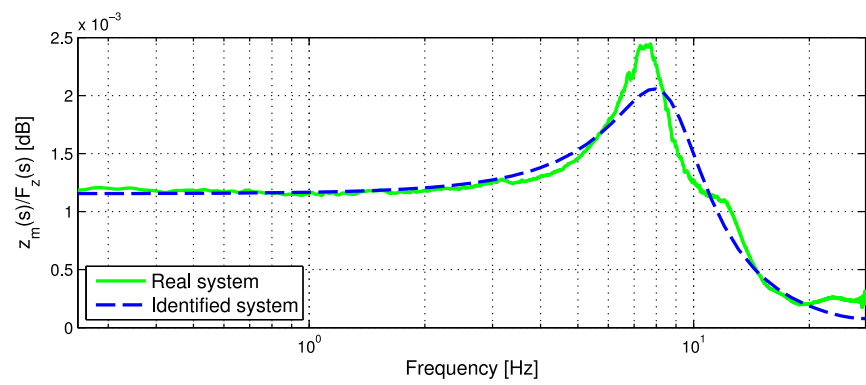

(c)

Fig. 5. Bode plot of the response of the real-system (solid green line) and the model with identified parameters (dashed blue line). 


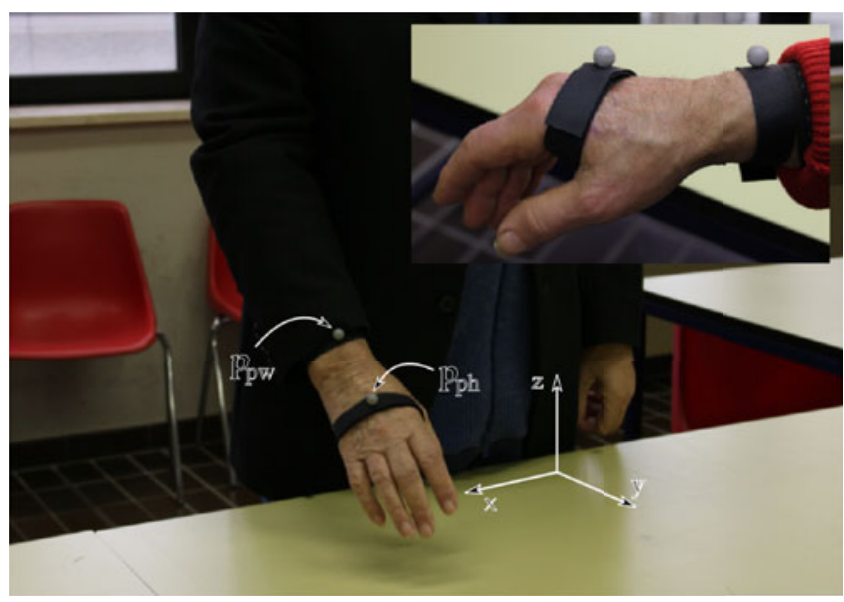

Fig. 6. The acquisition scenario; spherical markers are used to track the positions $\mathbf{p}_{p w}$ and $\mathbf{p}_{p h}$.

are respectively shown in Figs. 5a, 5b, and 5c. Explicit form of Eq. (1) is given for the $x$ axis in Eq. (4) in Appendix A.

\section{Registration Phase}

As mentioned in the introduction section, the system that is proposed in this paper is controlled by playing back trajectories that are acquired on patients suffering from tremor disease. In this section, we describe the procedure that was adopted for acquiring and analysing such trajectories.

In order to narrow the focus of our work, we decided to concentrate on parkinsonian tremor. We examined and acquired data from six patients with the same disease with different degrees of severity of tremor on upper limbs. Among them we selected three sets of data for further analysis, taken from three different patients that were representative of mild, medium and high degree of severity of the impairment. The recording session took place in one day at the faculty of engineering of University of Ancona.

\subsection{Data Acquisition and Analysis}

A marker-based wireless tracking device OptiTrack Trio [22] was employed for the acquisition of hand trajectories. This system was chosen thanks to the following attributes:

- its resolution, accuracy and sampling rate are in line with the requirements for the acquisition of typical expected trajectories;

- it shows large workspace and extremely low intrusiveness: markers to be worn are small/lightweight spheres that can be attached to the patient's body through simple elastic strips;

- it is a portable system that can be easily installed in an unstructured environment.

The acquisition is conducted for the right hand using two optical markers. The first is fixed on the dorsal side of the wrist and the second in the middle of the back the hand (see Fig. 6). Positions of the wrist-marker $\mathbf{p}_{p w}=\left[x_{p w}, y_{p w}, z_{p w}\right]$ and hand-marker $\mathbf{p}_{p h}=\left[x_{p h}, y_{p h}, z_{p h}\right]$ are acquired, where the three components of the vectors correspond to the Cartesian axes directions along which the device was previously characterised (see Section 3).

During the recording phase the patient kept her/his right hand relaxed in front of him with the elbow extended, at

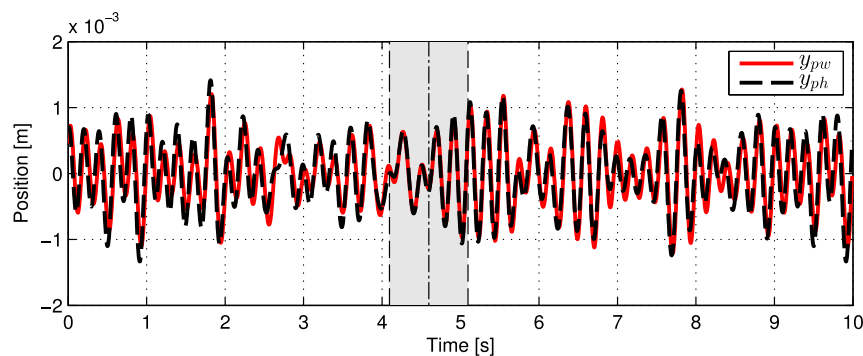

(a)

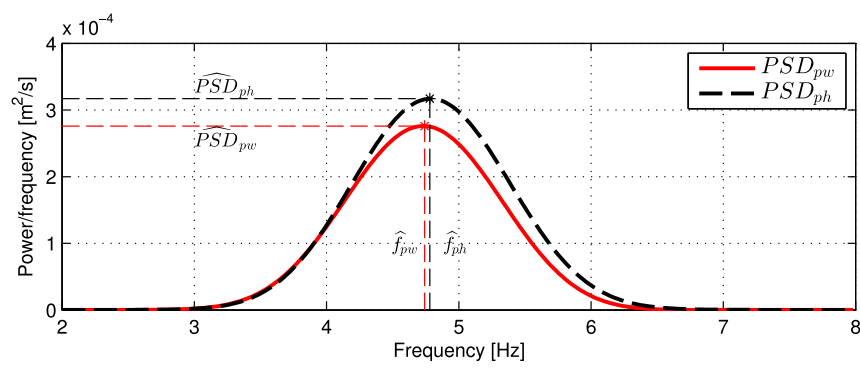

(b)

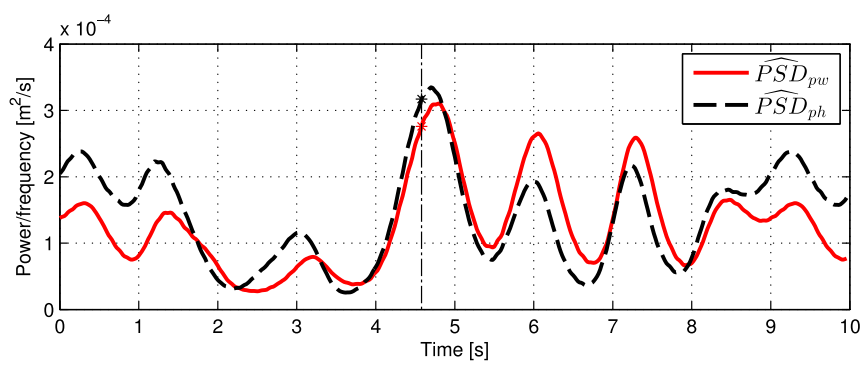

(c)

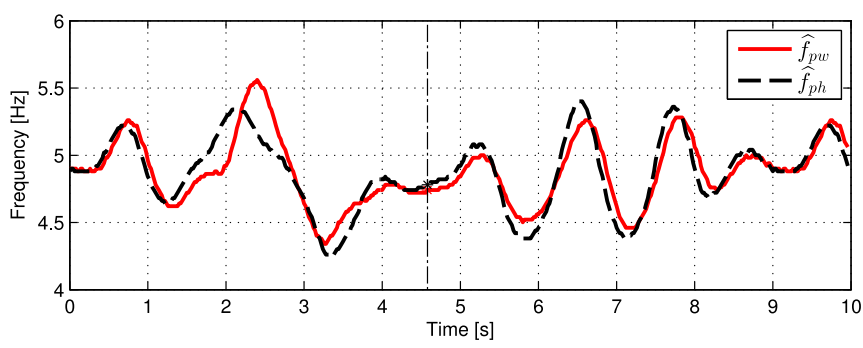

(d)

Fig. 7. (a) The recorded signal for patient $\mathrm{P} 1$ referred to the wrist and hand along the $y$ axis. (b) Its Power Spectral Density calculated in the time-window of $1 \mathrm{~s}$ (b). (c) The maximum value of the Power Spectral Density of the recorded signal versus time. (d) The main frequency of the Power Spectral Density of the recorded signal versus time.

approximately $5 \mathrm{~cm}$ from the table (Fig. 6). This reference posture is assumed by both the patient, during this acquisition phase, and by the healthy subject (the user) during the operation (see Section 6). Signals were acquired at $120 \mathrm{~Hz}$ for $60 \mathrm{~s}$ and filtered with a band-pass filter, in order to remove the low and high frequency contents which are not relevant for tremor simulation. Specifically, each component of $\mathbf{p}_{p w}$ and $\mathbf{p}_{p h}$ was filtered using a Chebyshev band-filter $\left(F_{b p}\right)$ of 2nd-order with normalised passband edge frequency from 4 to $12 \mathrm{~Hz}$ and $0.1 \mathrm{~dB}$ of peak-to-peak ripple in the passband obtaining respectively $\mathbf{p}_{p w}^{*}$ and $\mathbf{p}_{p h}^{*}$.

Fig. 7a shows an example of the time plot of the acquired and filtered signals. As shown, an oscillatory trajectory can be 
TABLE 2

Ranges of the Peak Value of the Power Spectral Density $\widehat{P S D}$ and the Corresponding

Frequency $\widehat{f}$ for the Patient's Hand and Wrist

\begin{tabular}{|c|c|c|c|c|c|}
\hline & & \multicolumn{2}{|c|}{ Wrist } & \multicolumn{2}{|c|}{ Hand } \\
\hline & & $\widehat{f}_{p w}(\mathrm{~Hz})$ & $\widehat{P S D}_{p w}\left(\frac{m}{s^{2}}\right)$ & $\widehat{f}_{p h}(\mathrm{~Hz})$ & $\widehat{P S D}_{p h}\left(\frac{m}{s^{2}}\right)$ \\
\hline \multirow[t]{3}{*}{ P1 } & $x$ & $4.4-6.2$ & $8.6 e^{-6}-9.7 e^{-4}$ & $4.3-6.1$ & $1.3 e^{-5}-3.4 e^{-3}$ \\
\hline & $y$ & $4.3-5.6$ & $2.7 e^{-5}-3.1 e^{-4}$ & $4.2-5.4$ & $2.6 e^{-5}-3.3 e^{-4}$ \\
\hline & $z$ & $4.6-5.8$ & $5.8 e^{-5}-4.1 e^{-3}$ & $4.6-6.0$ & $2.8 e^{-5}-2.0 e^{-3}$ \\
\hline \multirow[t]{3}{*}{ P2 } & $x$ & $3.5-5.5$ & $2.4 e^{-4}-2.8 e^{-3}$ & $3.0-5.5$ & $1.4 e^{-4}-1.8 e^{-3}$ \\
\hline & $y$ & $3.9-5.6$ & $2.4 e^{-4}-3.9 e^{-3}$ & $4.6-5.6$ & $7.3 e^{-4}-8.0 e^{-3}$ \\
\hline & $z$ & $3.9-5.8$ & $2.8 e^{-4}-6.8 e^{-3}$ & $4.2-5.7$ & $1.1 e^{-3}-2.2 e^{-2}$ \\
\hline \multirow[t]{3}{*}{ P3 } & $x$ & $3.5-4.8$ & $2.4 e^{-3}-2.0 e^{-2}$ & $2.0-4.3$ & $2.3 e^{-3}-3.8 e^{-2}$ \\
\hline & $y$ & $3.4-4.2$ & $4.7 e^{-4}-5.2 e^{-3}$ & $3.1-4.7$ & $1.4 e^{-3}-9.7 e^{-3}$ \\
\hline & $z$ & $3.5-4.3$ & $3.0 e^{-4}-6.1 e^{-3}$ & $3.5-4.8$ & $4.4 e^{-3}-3.2 e^{-2}$ \\
\hline
\end{tabular}

observed on both the wrist (solid red line) and the hand (dashed black line) of the patient's hand. In order to identify useful parameters, the acquired signals were elaborated through spectral analysis. Fig. $7 \mathrm{~b}$ shows the spectral response calculated on a time-window of $1 s$, chosen as a compromise between the richness of the frequency content of the output and quality of the estimation. Two parameters, hereafter referred as spectral parameters, that characterise the shape of the spectrum can be evaluated at each instant (see Fig. 7b):

1) $\widehat{P S D}$ : the peak value of the power spectral density which is a parameter that provides an insight of the intensity of the oscillating signal;

2) $\widehat{f}$ : the value of frequency registered in correspondence of $\widehat{P S D}$.

Figs. $7 \mathrm{c}$ and $7 \mathrm{~d}$ provide an example of the time plot of the estimated $\widehat{f}$ and $\widehat{P S D}$ for the wrist (solid red line) and hand (dashed black line) positions. As it can be observed these two parameters are variable over time. Table 2 reports the range of variation of $\widehat{f}$ and $\widehat{P S D}$ along all the Cartesian components for the three considered patients $(\mathrm{P} i)$ both for wrist and hand signals.

The three chosen patients show quite different degree of severity of hand tremor going from mild intensity for P1, to medium for P2 and severe tremors for P3. Oppositely, frequency ranges are higher for $\mathrm{P} 1$, intermediate for $\mathrm{P} 2$ and lower for P3.

\section{HAND-Tremor Controller}

The aim of the controller is to impose a tremor to the hand of the healthy users, which is an accurate reproduction of the tremor that is previously recorded on Parkinsonian patients, reminding that, in the prospected application, we need to leave the user able to decide her/his wanted movement without constrains.

The proposed controller relies on a simple architecture based on recording and playback. Specifically, the patient wrist trajectory, that is recorded and filtered according to the procedure described in the previous section, is superimposed on the natural goal-directed movements of the healthy user with the aim of inducing a similar tremor at hand level. One of the filtered trajectory $\mathbf{p}_{p w}^{*}(t)$ can be chosen among those acquired from different patients and employed as reference trajectory for the induced tremor at the level of the wrist.

It is important to underline that behind this control strategy a basic assumption is made, i.e., if we impose to the wrist of a healthy person the same trajectory of the wrist of a tremor-affected subject, the generated hand-tremor in the healthy subject is comparable to the hand-tremor of the disabled subject. This assumption is verified a posteriori in Section 6.

The simulation of hand tremor, with the described set up, can be implemented according to different strategies. In our previous work [8], we have tested three different controllers using artificially generated trajectories, and concluded that a component of adaptation is contributing to improve the behaviour of the system.

Assuming the dynamic model presented in Fig. 4, we propose here a further advancement in the controller that is based on an on-line estimation of the parameter $m_{h}$, i.e., the equivalent mass of the user arm.

The scheme of the controller is shown in Fig. 8 for the $x$ direction. The desired trajectory $x_{p w}^{*}$, is obtained by filtering the recorded trajectory $x_{p w}$ through the band-pass filter $F_{b p}(s)$ described in the previous section; $x_{p w}^{*}$ is fed into a system compensator, where its is multiplied by the global system impedance $Z_{\text {sys }}(s)$ to obtain the controller force $F_{x}$.

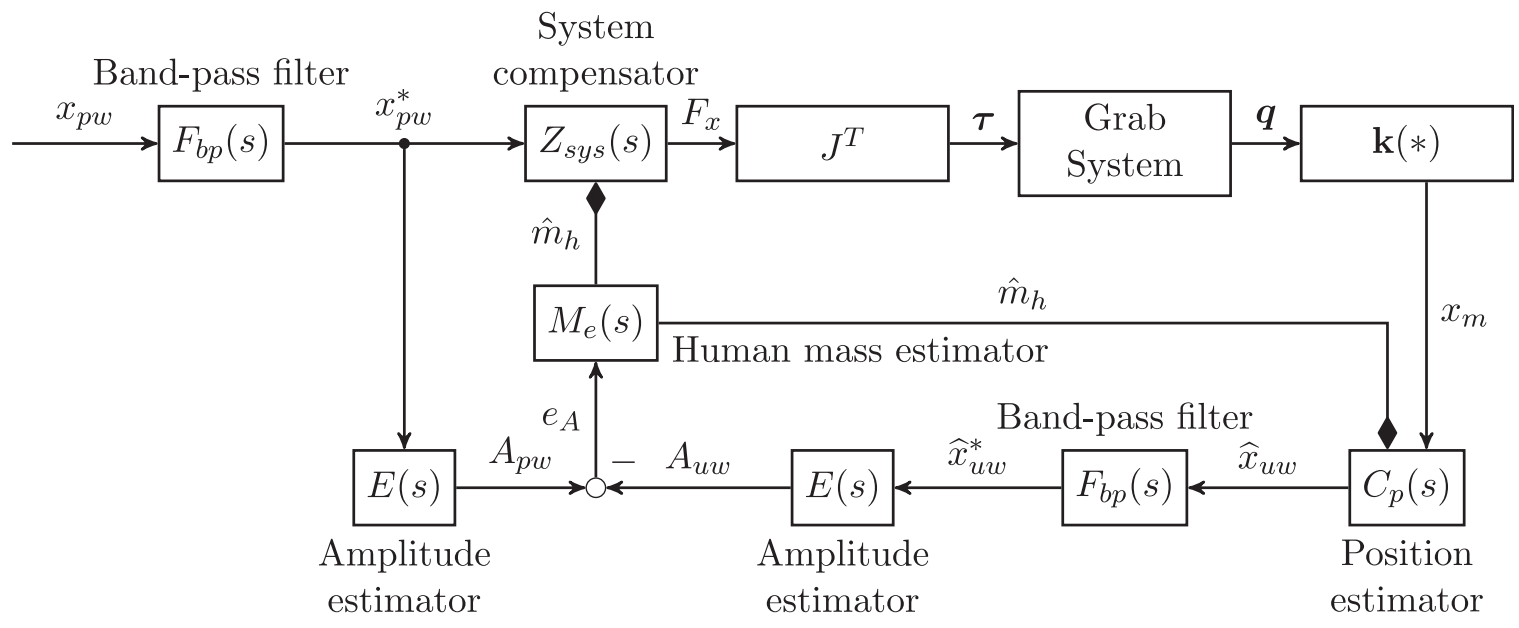

Fig. 8. Scheme of the adaptive controller. 
$Z_{\text {sys }}(s)$ is the transfer function of the system between $x_{m}$ and $F_{x}$ obtained by analysis of dynamic system represented in Fig. 4 (see explicit form in Eq. (6) in Appendix A). The parameters of this transfer function are shown in Table 1 featuring different values for each axis. The parameter $\hat{m}_{h}$ is adaptive and is updated through an estimation process described in the following. Its initial value is chosen in a range between 0.1 and $0.5 \mathrm{~kg}$.

The commanded motor-torques $\tau$ are calculated from $\mathbf{F}=\left[F_{x}, F_{y}, F_{z}\right]$ through differential kinematic transformation. The motor position reduced to Cartesian coordinate $\left(x_{m}, y_{m}, z_{m}\right)$ is calculated through direct kinematics from measured motor positions q. The estimated position of the user wrist $\widehat{x}_{u w}$ is obtained by the estimator $C_{p}(s)$, that is a linear filter that estimates the position of wrist of the user using the Cartesian position of the motor $x_{m}$ and the estimation of the user's arm mass $\widehat{m}_{h}$ (Eq. (5) in Appendix A). This filter is implemented through the transfer function that represents the system shown in Fig. 4, assuming as with input $x_{m}$ and as output $x_{w}$, and considering the dynamic parameters taken from the Table 1.

The estimated position is band-pass filtered through the same $F_{b s}(s)$ (obtaining $\widehat{x}_{u w}^{*}$ ) and employed by an amplitude estimator $E(s)$ that calculates its RMS amplitude on a time window of $1 s\left(A_{u w}\right)$, i.e., the actual amplitude of the vibration of the wrist. The actual estimated amplitude error is then calculated subtracting the desired amplitude $A_{p w}$ obtained calculating the RMS amplitude of the desired trajectory $x_{p w}^{*}$.

An updated value of the estimated user mass $\widehat{m}_{h}$ is obtained by the mass estimator $M_{e}(s)$, assuming that the error in amplitude is solely due to the variation of the mass of the user $m_{h}$. Actual value of the mass is then fed back into the system impedance $Z_{\text {sys }}(s)$ and into the position estimator $C_{p}(s)$.

$M_{e}(s)$ is a linear PID filter, with the error of amplitude between two signals $A_{p w}$ and $A_{w}$ in input, and with an estimation of mass $m_{h}$ as output that can assume values between 0.1 and $5 \mathrm{~kg}$. The calibration of the internal parameters is made heuristically. It is worth to notice, that the developed controller imposes a variation of a parameter of a LTI system (i.e., the estimated mass) thus could appear as formally inconsistent. However, since the dynamics of $\widehat{m}_{h}$ is consistently slower if compared with the dynamic of $x_{p w}^{*}$, it is still possible to treat the system with LTI approach and write the transfer functions $Z_{\text {sys }}(s)$ and $C_{p}(s)$.

In summary, we have implemented a controller that imposes forces, needed to generate the tremor on the user hand, calculated in open-loop on the base a recorded trajectory and through a model of the system dynamics. The RMS value of the generated tremor is instantaneously compared to the desired tremor intensity (calculated on the recorded trajectories). The error in the amplitude of tremor is fed to a PID filter which changes the model parameters with the aim of adjusting amplitude errors. Specifically, we assume that the error $e_{A}$ is only due to the difference between the estimated and real masses of the user. We are not considering to compensate possible error in frequency assuming that they can be neglected. All these assumptions will be verified a posteriori by analysing the performances of the controller.

The described controller was implemented along the three directions using different desired trajectories $x_{p w}, y_{p w}, z_{p w}$.

\section{Experimental Test AND ANALysis}

In this section, results of functional tests are reported in order to assess the capability of the system to reproduce the recorded trajectories.

The experimental scenario that was considered for the acquisition on patients described in Section 4.1 has been replicated for the tests. The arm of subject (healthy user) was connected to the GRAB assuming a posture that was closed to the reference posture of patients during the acquisition. No particular measures were taken to verify the exact match of the posture except for a visual inspection of angular position of articular joints of shoulder and elbow. The location of the user was chosen in a way that in the reference posture the HI was in the centre of the workspace (i.e., the point in which the device was dynamically characterised).

Tests were conducted on a total four users (three males and one female) in an age-range of 26-36 years old, using the acquired trajectories described in Section 4.1. During the tests, we used the same wireless tracking system (OptiTrack Trio) that was employed for the acquisition on patients, for the direct measuring of actual user's wrist $\left(\mathbf{p}_{u w}\right)$ and hand $\left(\mathbf{p}_{u h}\right)$ positions. Note that the readings from this tracker are not employed in the control loops. This optical tracker it is not going to be used in the final envisaged application but was only employed in this test to make it possible to verify the performance of the system. The markers were mounted in the same positions used for the acquisition on patients described in Section 4.1.

The $\widehat{P S D}$ and $\widehat{f}$ calculated on the generated trajectories has been considered as an evaluation/comparison parameter, i.e., the plots of the PSD of the recorded trajectories on Parkinson's patients and of the trajectories obtained for user's hand with the haptic system are compared.

Figs. 9a and $9 \mathrm{~b}$ show respectively an example of the time plot of the $\widehat{P S D}$ and the and $\widehat{f}$ for the wrist of the patient (solid red line) and of the user (dashed blue line). Figs. 9c and $9 \mathrm{~d}$ show respectively, for the same example, the time plot of the $\widehat{P S D}$ and the and $\widehat{f}$ for the hand of the patient (solid black line) and of the user (dashed blue line).

The Fig. 10 shown the estimated mass dynamics during one of the tests showing its reduced rate of variation. In the reported case, the initial value of $\hat{m}_{h}$ was set to one of the chosen boundary values (i.e., $0.1 \mathrm{~kg}$ ).

In order to evaluate the performance of the controller, it is useful to consider the maximum relative error that is committed in tracking the $\widehat{P S D}$ and the and $\widehat{f}$ over all the acquisition time, which are respectively defined as:

$$
\begin{gathered}
e_{S *}=\frac{\max _{0<t<T}\left|\left(\widehat{P S D}_{u *}-\widehat{P S D}_{p *}\right)\right|}{\max \left(\widehat{P S D}_{p *}\right)} \\
e_{f *}=\frac{\max _{0<t<T}\left|\left(\widehat{f}_{u *}-\widehat{f}_{p *}\right)\right|}{\max \left(\widehat{f}_{p *}\right)},
\end{gathered}
$$

where $T$ is acquisition time of the test, $P S D_{u *}$ and $P S D_{p *}$ are respectively the instantaneous maximum value of the spectral density for the user and for the reference trajectory of 


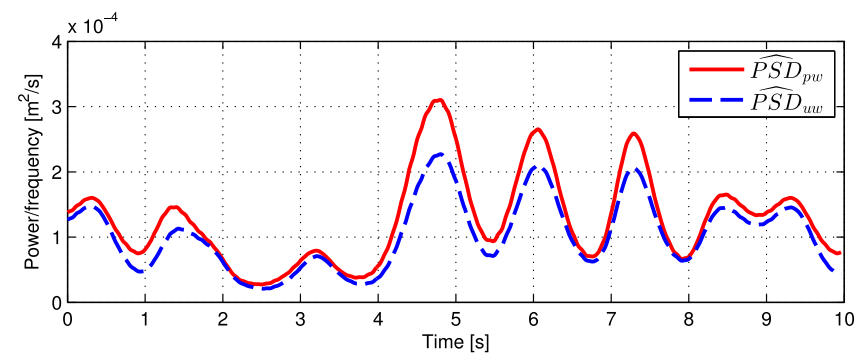

(a)

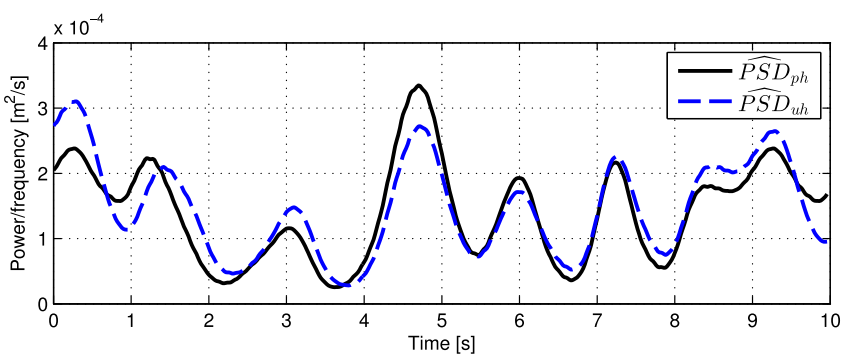

(c)

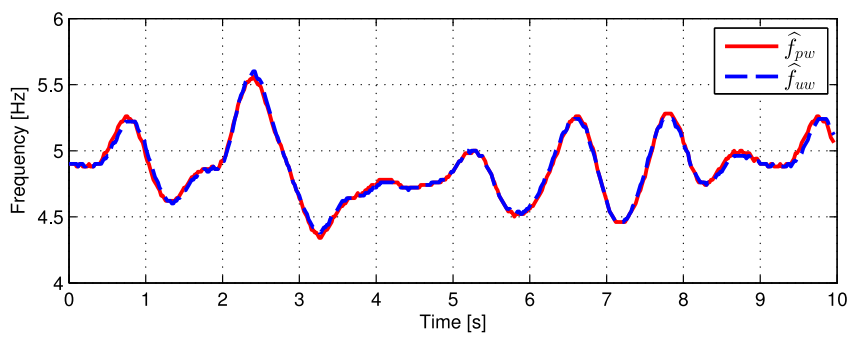

(b)

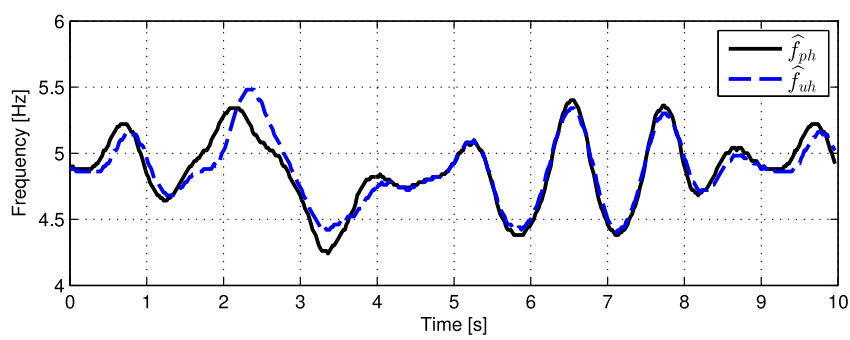

(d)

Fig. 9. Examples of plot of the power spectrum of the system response during the tests with the users. Subscripts in the legends read as: $p w \rightarrow$ patient's wrist, $u w \rightarrow$ user's wrist, $p h \rightarrow$ patient's hand, $u h \rightarrow$ user's hand.

the patient, while $f_{u *}$ and $f_{p *}$ are the corresponding frequency for the user and for the reference trajectory of the patient. The symbol $*$ become $w$ is the error is referred to wrist trajectory and $h$ for the hand.

The Table 3 resumes the tracking error for the four subjects that are using the system when the three reference signals corresponding to mild (patient P1), medium (patient P2) and strong (patient P3) tremor severity are imposed. From the results that are shown we can observe that a good tracking is obtained at wrist level with a very low error on frequency in the range of $0 \%<e_{f w} \leq 1 \%$ and on peak of PSD in the range of $3 \% \leq e_{S w} \leq 16 \%$.

As expected, greater errors are estimated for the hand tracking, with a maximum error on frequency of $e_{f h} \leq 1.9 \%$ and a range of error on peak of PSD $4.2 \% \leq e_{S h} \leq 21 \%$. Such errors are considered more than acceptable having in mind that the final aim of the device that is to provide a sensation of tremor. This makes it possible to conclude that the assumed hypotheses are reasonably valid.

It is worth noticing that the good match between the commanded and generated spectrum of tremor at the hand level is obtained in quite controlled conditions that impose several limitations in the application. Specifically, in order for the considered experimental conditions to be valid also in the real application scenario the following limitations should be considered:

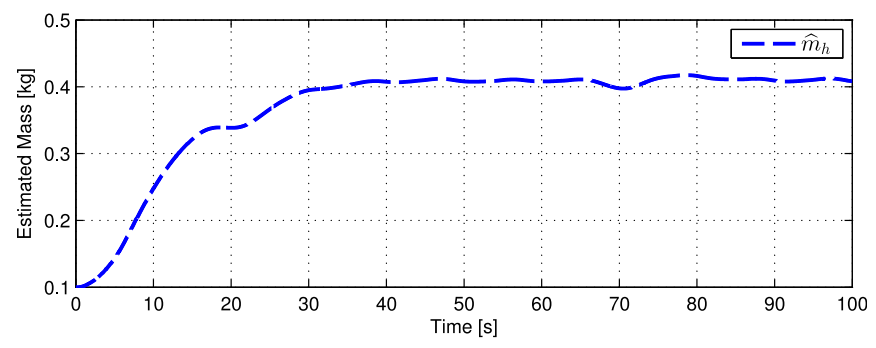

Fig. 10. The estimated mass $\widehat{m}_{h}$.
- the user is asked to keep his arm posture maintaining his hand close to the initial position: this limitation allows the user to perform only limited types of manipulative tasks;

- the user should maintain his muscles quite relaxed limiting the allowable manipulative actions to those which do not require strong muscular activity.

As previously underlined, these appear as very strong limitations. However, several simple tests can be done even with such limitations. For example, the device was preliminarily employed in an engineering design experiment in which designers of kitchen appliances from the company INDESIT S.r.l. were asked to use the developed haptic tool [23]. The system was employed in a design experiment of kitchen gas hobs and gas switch. Designers were asked to interact with a set of existing gas hob and gas switches with the device worn and controlled with different levels of tremor imposed. After this test sessions, the designers were asked to formulate new design for the gas controllers which takes into account the experienced interactive experiment. Interesting design alternatives were proposed.

\section{Conclusions}

A haptic interface was successfully employed in the task of simulating/inducing on healthy subjects a hand-tremor that is similar to that observed on subject with certain types neurological syndrome. This tool is developed for designers that are asked to conceive products and services that have to be accessible for all. The scope is to induce the user (i.e., the designer) to feel in first person the effects of the disease and get empathy with the end user of her/his products.

A desktop haptic interface with a large workspace was employed to induce controlled vibrations on the hand of a healthy user to simulate the effect of hand-tremor disability when manipulating and handling objects.

A specially designed lightweight spherical joint was studied to connect the haptic device to the forearm and 
TABLE 3

Maximum Spectral Error

\begin{tabular}{|c|c|c|c|c|c|c|c|c|c|c|c|c|c|c|c|c|c|}
\hline & \multicolumn{4}{|c|}{ U1 } & \multicolumn{4}{|c|}{ U2 } & \multicolumn{4}{|c|}{ U3 } & \multicolumn{4}{|c|}{ U4 } \\
\hline & & \multicolumn{2}{|c|}{ Wrist } & \multicolumn{2}{|c|}{ Hand } & \multicolumn{2}{|c|}{ Wrist } & \multicolumn{2}{|c|}{ Hand } & \multicolumn{2}{|c|}{ Wrist } & \multicolumn{2}{|c|}{ Hand } & \multicolumn{2}{|c|}{ Wrist } & \multicolumn{2}{|c|}{ Hand } \\
\hline & & $e_{f w}$ & $e_{S w}$ & $e_{f h}$ & $e_{S h}$ & $e_{f w}$ & $e_{S w}$ & $e_{f h}$ & $e_{S h}$ & $e_{f_{w}}$ & $e_{S w}$ & $e_{f h}$ & $e_{S h}$ & $e_{f w}$ & $e_{S w}$ & $e_{f h}$ & $e_{S h}$ \\
\hline & & $\%$ & $\%$ & $\%$ & $\%$ & $\%$ & $\%$ & $\%$ & $\%$ & $\%$ & $\%$ & $\%$ & $\%$ & $\%$ & $\%$ & $\%$ & $\%$ \\
\hline \multirow[t]{3}{*}{ P1 } & $x$ & $<1$ & 8.1 & $<1$ & 4.6 & $<1$ & 12 & $<1$ & 17 & $<1$ & 8.8 & $<1$ & 12 & $<1$ & 8.5 & $<1$ & 13 \\
\hline & $y$ & $<1$ & 7.8 & 1.3 & 8.2 & $<1$ & 9 & 1.8 & 19 & $<1$ & 4.3 & $<1$ & 11 & $<1$ & 15 & $<1$ & 16 \\
\hline & $z$ & $<1$ & 3 & $<1$ & 4.1 & $<1$ & 4.8 & $<1$ & 16 & $<1$ & 8.5 & $<1$ & 17 & $<1$ & 3 & $<1$ & 15 \\
\hline \multirow[t]{3}{*}{ P2 } & $x$ & $<1$ & 8.8 & 1.2 & 14 & $<1$ & 15 & $<1$ & 17 & $<1$ & 5 & $<1$ & 4.1 & $\sim 1$ & 7.3 & $\sim 1$ & 5.8 \\
\hline & $y$ & $<1$ & 14 & $<1$ & 13 & $<1$ & 14 & $<1$ & 21 & $<1$ & 9.6 & $<1$ & 21 & $<1$ & 14 & $<1$ & 16 \\
\hline & $z$ & $<1$ & 14 & $<1$ & 14 & $<1$ & 11 & $<1$ & 16 & $<1$ & 7.7 & $<1$ & 16 & $<1$ & 1.9 & $<1$ & 16 \\
\hline \multirow[t]{3}{*}{ P3 } & $x$ & $<1$ & 7.4 & $\sim 1$ & 13 & $<1$ & 12 & 1.9 & 17 & $<1$ & 2.6 & $\sim 1$ & 11 & $<1$ & 5.4 & $<1$ & 9.2 \\
\hline & $y$ & $<1$ & 14 & 1.5 & 19 & $<1$ & 8.5 & $<1$ & 11 & $<1$ & 11 & $<1$ & 13 & $<1$ & 15 & $<1$ & 15 \\
\hline & $z$ & $<1$ & 9.5 & $<1$ & 16 & $<1$ & 3.8 & $<1$ & 18 & $<1$ & 16 & $<1$ & 18 & $\sim 1$ & 15 & $\sim 1$ & 16 \\
\hline
\end{tabular}

effectively transmitting the needed oscillating forces. The setup allows the user to interact with real objects while the haptic interface introduces vibratory movements that superimpose on the voluntary actions.

A campaign of experimental acquisition was realised on a set of patients affected by tremor diseases with the aim of acquiring trajectories to be employed as an input for the developed system.

An adaptive controller for the haptic interface was studied, implemented and tested to allow the user to program the typology of induced tremor. The controller was conceived and designed in order to minimise disturbances on low-frequency voluntary motions and to robustly control the amplitude of vibration of the user's wrist.

Future works will be focussed on the improvement of the current system extending the functionalities to the whole workspace of the haptic device. In parallel, tests should be designed and conducted with the support of medical doctors to evaluate manipulative performances of patients affected by tremor and compare to those of users with simulated hand-tremor.

\section{APPENDIX A}

\section{TRANSFER FUNCTION EQUATION}

\section{A.1 GRAB Identification Transfer Function}

Transfer function of the grab system during the identification phase, for the $x$ axis:

$$
\frac{x_{m}(s)}{F_{x}(s)}=\frac{\frac{m_{m}}{m_{m} m_{l}} s^{2}+s \frac{c_{t}+c_{c}}{m_{m} m_{l}}+\frac{k_{t} k_{c}}{m_{m} m_{l}}}{s^{4}+s^{3} A_{g}+s^{2} B_{g}+s C_{g}+D_{g}} ;
$$

with:

$$
\begin{aligned}
A_{g} & =\frac{c_{t} m_{l}+c_{c} m_{l}+m_{m} c_{t}}{m_{m} m_{l}}, \\
B_{g} & =\frac{k_{c} m_{l}+c_{c} c_{t}+k_{t} m_{l}+m_{m} k_{t}}{m_{m} m_{l}}, \\
C_{g} & =\frac{k_{c} c_{t}+c_{c} k_{t}}{m_{m} m_{l}}, \\
D_{g} & =\frac{k_{c} k_{t}}{m_{m} m_{l}} .
\end{aligned}
$$

\section{A.2 Position Estimator}

Transfer function of $C_{p}(s)$ :

$$
C_{p}(s)=\frac{s^{2} \frac{c_{c} c_{t}}{m_{m} \tilde{m}_{h}}+s \frac{k_{c} c_{t}+c_{c} k_{t}}{m_{m} \hat{n}_{h}}+\frac{k_{c} k_{t}}{m_{m} \hat{m}_{h}}}{s^{4}+s^{3} A_{c}+s^{2} B_{c}+s C_{c}+D_{c}}
$$

with:

$$
\begin{aligned}
A_{c} & =\frac{c_{t} \hat{m}_{h}+c_{c} \hat{m}_{h}+c_{c} m_{m}}{m_{m} \hat{m}_{h}}, \\
B_{c} & =\frac{k_{c} \hat{m}_{h}+m_{m} k_{c}+c_{c} c_{t}+\hat{m}_{h} k_{t}}{m_{m} \hat{m}_{h}}, \\
C_{c} & =\frac{k_{c} c_{t}+c_{c} k_{t}}{m_{m} \hat{m}_{h}}, \\
D_{c} & =\frac{k_{c} k_{t}}{m_{m} \hat{m}_{h}} .
\end{aligned}
$$

\section{A.3 System Compensator}

Transfer function of $Z_{\text {sys }}(s)$ :

$$
Z_{s y s}(s)=\frac{K_{z}\left(s^{4}+s^{3} A_{z}+s^{2} B_{z}+s C_{z}+D_{z}\right)}{s^{4}+s^{3} E_{z}+s^{2} F_{z}+s G_{z}+H_{z}} ;
$$

with:

$$
\begin{aligned}
K_{z} & =\frac{p^{2} m_{m} \hat{m}_{h} m_{l}}{c_{t} c_{c}}, \\
A_{z} & =\frac{\hat{m}_{h} c_{t}\left(m_{l}+m_{m}\right)+m_{l} c_{c}\left(\hat{m}_{h}+m_{m}\right)}{m_{m} \hat{m}_{h} m_{l}}, \\
B_{z} & =\frac{m_{l} k_{c}\left(\hat{m}_{h}+m_{m}\right)+c_{c} c_{t} m_{T}+\hat{m}_{h} k_{t}\left(m_{l}+m_{m}\right)}{m_{m} \hat{m}_{h} m_{l}}, \\
C_{z} & =\frac{c_{t} k_{c} m_{T}+c_{c} k_{t} m_{T}}{m_{m} \hat{m}_{h} m_{l}}, \\
D_{z} & =\frac{k_{c} k_{t} m_{T}}{m_{m} \hat{m}_{h} m_{l}}, \\
E_{z} & =\frac{2 p c_{c} c_{t}+c_{t} k_{c}+c_{c} k_{t}}{c_{t} c_{c}}, \\
F_{z} & =\frac{p^{2} c_{c} c_{t}+2 p\left(c_{t} k_{c}+c_{c} k_{t}\right)+k_{c} k_{t}}{c_{t} c_{c}}, \\
G_{z} & =\frac{p^{2}\left(c_{t} k_{c}+c_{c} k_{t}\right)+2 p k_{c} k_{t}}{c_{t} c_{c}}, \\
H_{z} & =\frac{p^{2} k_{c} k_{t}}{c_{t} c_{c}}, \\
m_{T} & =\hat{m}_{h}+m_{l}+m_{m},
\end{aligned}
$$


where the two poles $\mathrm{p}$ are introduced in order to guarantee the causality of the transfer functions.

\section{ACKNOWLEDGMENTS}

This research is partially funded by the European project VERITAS-Virtual and Augmented Environments and Realistic User Interactions to achieve Embedded Accessibility DesignS (Project No. 247765), 7th Framework Programme, Theme FP7-ICT-2009.7.2, Accessible and Assistive ICT.

\section{REFERENCES}

[1] D. McDonagh, "Do it until it hurts! Empathic design research," Des. Principles Practices: An Int. J., vol. 2, no. 3, pp. 103-109, 2008.

[2] C. Cardoso and P. Clarkson, "Simulation in user-centred design: Helping designers to empathise with atypical users," J. Eng. Des., vol. 23, no. 1, pp. 1-22, 2012.

[3] (2015, Dec.). AGNES - Age Gain Now Empathy System [Online]. Available: http://agelab.mit.edu/agnes-age-gain-now-empathysystem

[4] T. Oikonomou, K. Votis, D. Tzovaras, and P. Korn, "An open source tool for simulating a variety of vision impairments in developing swing applications," in Proc. 5th Int. Conf. Universal Access Human-Comput. Interaction. Addressing Diversity, 2009, pp. 135-144.

[5] C. Strobbe, B. Frees, and J. Engelen, "An accessibility checker for libreoffice and openoffice. org writer," in Proc. 13th Int. Conf. Comput. Helping People with Special Needs, 2012, pp. 484-491.

[6] K. Breiner, T. Wüchner, and M. Brunnlieb, "The Disabilitysimulator: Simulating the influences of disabilities on the usability of graphical user interfaces," in Proc. 11th Int. Conf. Ergonomics Health Aspects Work with Comput., 2011, pp. 109-118.

[7] C. Avizzano, S. Marcheschi, M. Angerilli, M. Fontana, M. Bergamasco, T. Gutierrez, and M. Mannegeis, "A multi-finger haptic interface for visually impaired people," in Proc. 12th IEEE Int. Workshop Robot Human Interactive Commun., 2003, pp. 165-170.

[8] G. P. Rosati Papini, M. Fontana, R. Vertechy, M. Carrozzino, and M. Bergamasco, "Haptic Hand-tremor simulation for enhancing empathy with disabled users," in Proc. RO-MAN, 2013, pp. 553-558.

[9] P. Crawford and E. E. Zimmerman, "Differentiation and diagnosis of tremor," Am. Family Phys., vol. 83, no. 6, p. 697, 2011.

[10] D. E. Vaillancourt and K. M. Newell, "The dynamics of resting and postural tremor in parkinson's disease," Clinical Neurophysiol., vol. 111, no. 11, pp. 2046-2056, 2000.

[11] G. Deuschl, R. Wenzelburger, K. Löffler, J. Raethjen, and H. Stolze, "Essential tremor and cerebellar dysfunction clinical and kinematic analysis of intention tremor," Brain, vol. 123, no. 8, pp. 1568-1580, 2000.

[12] S. Morrison and K. M. Newell, "Postural and resting tremor in the upper limb," Clinical Neurophysiol., vol. 111, no. 4, pp. 651-663, 2000.

[13] K. Wyne, "A comprehensive review of tremor," Jaapa, vol. 18, no. 12, pp. 43-50, 2005.

[14] M. Gresty and D. Buckwell, "Spectral analysis of tremor: Understanding the results," J. Neurol., Neurosurgery Psychiatry, vol. 53, no. 11, pp. $976-981,1990$.

[15] C. Avizzano and M. Bergamasco, "Technological aids for the treatment of tremor," in Proc. 6th Int. Conf. Rehab. Robot., 1999, pp. 261-267.

[16] C. N. Riviere, W. T. Ang, and P. K. Khosla, "Toward active tremor canceling in handheld microsurgical instruments," IEEE Trans. Robot. Autom., vol. 19, no. 5, pp. 793-800, Oct. 2003.

[17] W. Latt, U.-X. Tan, C. Shee, and W. Ang, "A compact Handheld active physiological tremor compensation instrument," in Proc. IEEE/ASME Int. Conf. Adv. Intell. Mechatronics, 2009, pp. 711-716.

[18] M. Lakie, C. A. Vernooij, T. M. Osborne, and R. F. Reynolds, "The resonant component of human physiological hand tremor is altered by slow voluntary movements," The J. Physiol., vol. 590, no. 10 , pp. 2471-2483, 2012.

[19] T. Flash, R. Inzelberg, E. Schechtman, and A. D. Korczyn, "Kinematic analysis of upper limb trajectories in parkinson's disease," Exp. Neurol., vol. 118, no. 2, pp. 215-226, 1992.
[20] S. Marcheschi, F. Salsedo, M. Fontana, F. Tarri, O. PortilloRodríguez, and M. Bergamasco, "High performance explicit force control for finger interaction haptic interface," in Proc. EuroHaptics Conf. Symp. Haptic Interfaces Virtual Environ. Teleoper. Syst., 2007, pp. $464-469$.

[21] M. Fontana, S. Marcheschi, F. Salsedo, and M. Bergamasco, "A Three-axis force sensor for dual finger haptic interfaces," Sensors (Switzerland), vol. 12, no. 10, pp. 13 598-13 616, 2012.

[22] (2015, Dec.). OptiTrack Trio, NaturalPoint, Inc. DBA OptiTrack [Online]. Available: http:/ /www.naturalpoint.com

[23] L. Boffi, M. Fontana, G. P. Rosati Papini, and M. Milani, "Supporting the designers to build empathy with people with parkinson's disease: The role of a hand tremor simulating device and of user research with end-users," in Proc. DRS: Design's Big Debates, 2014, pp. 1526-1534.

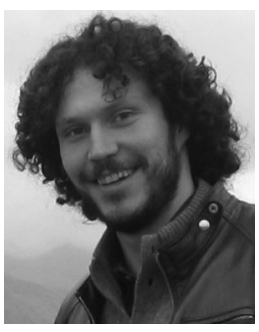

Gastone Pietro Rosati Papini received the master's degree in automation engineering (with honours) at the University of Pisa in 2011. Currently, he is working toward the $\mathrm{PhD}$ degree in emerging digital technologies at PERCRO Lab, Scuola Superiore Sant'Anna. His main research interests concern the modelling and control of complex mechatronic systems.

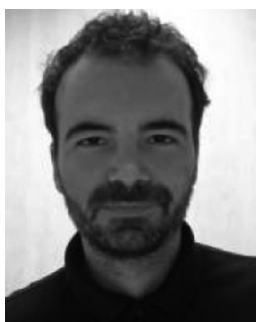

Marco Fontana received the MSc degree in mechanical engineer in 2003 from the University of Pisa and the PhD degree in engineering in 2008 from Scuola Superiore Sant'Anna. He is an assistant professor of mechanics of machine at Scuola Superiore Sant'Anna. His research focusses on the design, modelling, and development of mechatronic systems for robotic, automation, and energy harvesting applications.

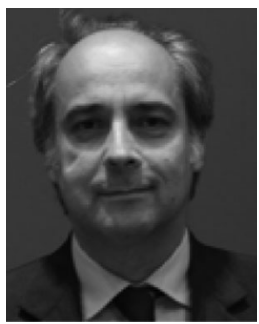

Massimo Bergamasco is a full professor of mechanics of machine at Scuola Superiore Sant'Anna since 2006. He has been a pioneer researchers in haptics in Europe. He has been responsible for several European projects and he is a member of the editorial board of several scientific journals and member of steering committee of several international conferences. His scientific activity includes more than 200 scientific papers published in journals and/or international conference proceedings.

$\triangleright$ For more information on this or any other computing topic, please visit our Digital Library at www.computer.org/publications/dlib. 